Кривов Максим Викторович, к.т.н., доцент, Ангарский государственный технический университет, e-mail:vmk@angtu.ru

МОДЕЛИРОВАНИЕ ОБРАТНЫХ ПОТОКОВ В ТРЕНАЖЕРНОЙ МОДЕЛИ

Krivov M.V.

\title{
SIMULATION OF REVERSE FLOWS IN THE TRANING MODEL
}

\begin{abstract}
Аннотация. Рассмотрены подходы к математическому моделированию реверсивных технологических потоков в тренажерных моделях компьютерных тренажерных комплексов.

Ключевые слова: компьютерный тренажерный комплекс, тренажерная модель, модель потоков.

Abstract. Approaches to mathematical modeling of reversible technological flows in training models of computer training complexes are considered.
\end{abstract}

Keywords: computer training complex, training model, flow mode.

Ориентирование потоков в математическом описании химикотехнологических систем (ХTC) является важным условием их корректного расчета. Расходы потоков определяют накопление и расход субстанций (веществ и энергии) в материальных и тепловых балансах моделируемого объекта.

В общем случае расход определяется выражением:

$$
F=K v \xi \sqrt{\left(P_{1}-P_{0}\right)},
$$

где $K v$ - коэффрициент пропускной способности потока; $\xi$ - коэффициент проходного сечения запорной арматуры и клапанов на потоке; $P_{0}$ - давление приёмника потока; $P_{l}$ - давление источника потока.

Из (1) вытекает условие, что поток в модели не может быть реверсивным из-за отрицательного значение под знаком радикала.

В связи с этим остановимся на одном важном обстоятельстве, характерном для тренажёрных моделей - обратном потоке. В технологических процессах он возникает, как правило, в режимах пуска или останова процесса, когда обратным ходом подают технологический поток из приемника в источник, например, для заполнения или опорожнения аппаратов.

Обратный поток, с одной стороны, изменяет структуру материального и теплового балансов, а, следовательно, и структуру математического описания XTC, но, с другой стороны, обратный поток присущ не каждому технологическому потоку (например, в силу физической невозможности его реализации или наличия обратного клапана).

Игнорирование обратных потоков повлечёт к нарушению принципа полноты математического описания и ограничению в моделировании технологических режимов ХТС тренажёрной моделью.

Возможно два пути решения данной проблемы. Первый - создание с каждой моделью прямого потока, модели обратного потока (рисунок 1). Тогда, с учетом (1), получаем: 


$$
\begin{gathered}
F \in F W_{0}=\left\{\begin{array}{c}
K v \xi \sqrt{\left(P_{1}-P_{0}\right)}, P_{1} \geq P_{0}, \\
0, P_{1}<P_{0}
\end{array},\right. \\
F \in F W_{0}^{R}=\left\{\begin{array}{c}
K v \xi \sqrt{\left(P_{0}-P_{1}\right)}, P_{0} \geq P_{1}, \\
0, P_{0}<P_{1}
\end{array}\right.
\end{gathered}
$$

где $F$ - расходы технологического потока, включённые в кортежи информационных потоков $F W_{0}$ и $F W_{0}^{R}$.

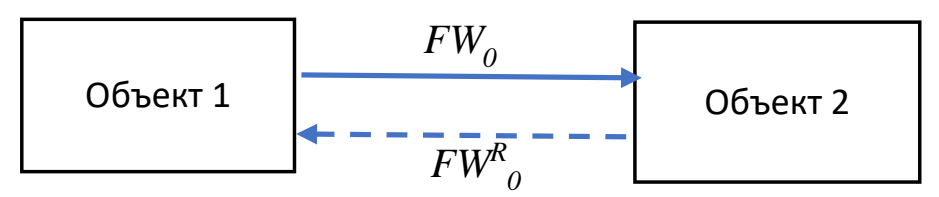

Рисунок 1. Создание дополнительного обратного потока

Критическим замечанием первого подхода является увеличение числа информационных моделей потоков и усложнение алгоритмов их обработки.

Второй подход состоит в разработке реверсивного информационного потока (рисунок 2), описываемого кортежем:

$$
F W_{i}=\left\langle T_{i}, P_{i}, C_{i}^{j}, \ldots, F_{i}^{f}, F_{i}^{b} \ldots\right\rangle
$$

где $T$ - температура технологического потока; $P$ - давление источника потока; $C_{j}$ - концентрации j-го компонента в потоке; $F_{i}^{f}, F_{i}^{b}$ - соответственно, численное значение расходов прямого и обратного технологического потока, включённых в кортеж данных $i$-го информационного потока $F W$.

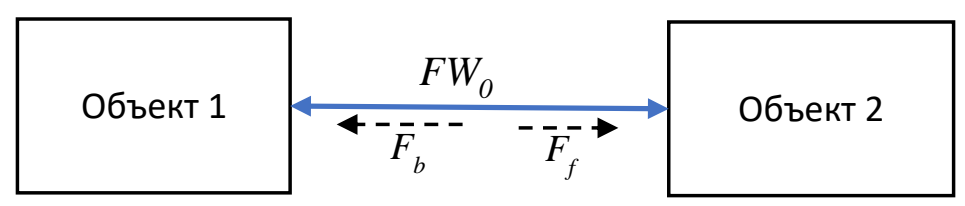

Рисунок 2. Создание реверсивного потока

Тогда для $i$-го информационного потока:

$$
\begin{gathered}
F_{f} \in F W_{i}=\left\{\begin{array}{c}
K v \xi \sqrt{\left(P_{1}-P_{0}\right)}, P_{1} \geq P_{0} \\
0, P_{1}<P_{0}
\end{array}\right. \\
F_{b} \in F W_{i}=\left\{\begin{array}{c}
K v \xi \sqrt{\left(P_{0}-P_{1}\right)}, P_{0} \geq P_{1} \\
0, P_{0}<P_{1}
\end{array}\right.
\end{gathered}
$$

Теперь, когда прямой и обратный расход являются элементами одного кортежа, материальный баланс будет иметь вид:

$$
\frac{d F W_{1} \rightarrow C_{j}}{d t}=\frac{F W_{0} \rightarrow F_{f} \cdot F W_{0} \rightarrow C_{j}-F W_{1} \rightarrow F_{f} \cdot F W_{1} \rightarrow C_{j}+F W_{1} \rightarrow F_{b} \cdot F W_{1} \rightarrow C_{j}}{V}+G(\ldots),
$$

где $G()$ - функция генерации субстанции; $t$ - время.

Достоинство данного подхода - число и содержание информационных потоков соответствует технологическим потокам, что облегчает процесс декомпозиции и понимание расчетной схемы тренажерной модели. 